\title{
Potassium-chelating drug sodium polystyrene sulfonate enhances lysosomal function and suppresses proteotoxicity
}

Cyrene Arputhasamy ${ }^{1}$, Mark Lucanic ${ }^{1}$, Anand Rane ${ }^{1}$, Minna Schmidt ${ }^{1,2}$, Theo Garett $^{1}$, Anna C. Foulger ${ }^{1}$, Michael Broussalian ${ }^{1,3}$, Elena Battistoni ${ }^{1,3}$, Rachel B. Brem $^{1,4}$, Gordon J. Lithgow ${ }^{1,2}$, Manish Chamoli ${ }^{1 \#}$, Julie K. Andersen ${ }^{1,2 \#}$

${ }^{1}$ Buck Institute for Research on Aging, Novato, CA, USA

2USC Leonard Davis School of Gerontology, University of Southern California, Los Angeles, CA, USA

${ }^{3}$ Dominican University of California, San Rafael, CA, USA

${ }^{4}$ Department of Plant and Microbial Biology, UC Berkeley, Berkeley, CA, USA

\#Correspondence to Manish Chamoli: mchamoli@buckinstitute.org and Julie K. Andersen: jandersen@buckinstitute.org

\section{HIGHLIGHTS}

- Sodium polystyrene sulfonate improves $\mathrm{pH}$-dependent processing of lysosomal cargo, enhances proteotoxic stress resistance and extends lifespan in C. elegans

- Sodium polystyrene sulfonate boosts lysosomal function in human neuronal cells and reduces level of aggregation-associated phosphorylated-TAU

\begin{abstract}
Lysosomes are crucial for degradation and recycling of damaged proteins and cellular components. Therapeutic strategies enhancing lysosomal function are a promising approach for aging and age-related neurodegenerative diseases. Here, we show that an FDA approved drug sodium polystyrene sulfonate (SPS), used to reduce high blood potassium in humans, enhances lysosomal function both in C.elegans and in human neuronal cells. Enhanced lysosomal function following SPS treatment is accompanied by the suppression of proteotoxicity caused by expression of the neurotoxic peptides $A \beta$ and TAU. Additionaly, treatment with SPS imparts health benefits as it significantly increases lifespan in C.elegans. Overall our work supports the potential use of SPS as a prospective geroprotective intervention.
\end{abstract}

\section{KEYWORDS}

C. elegans, lysosome, autophagy, potassium restriction, lifespan, proteotoxicity, $\mathrm{SH}-$ SY5Y neuronal cells, amyloid- $\beta$, neurodegeneration 


\section{INTRODUCTION}

Lysosomal dysfunction is associated with aging and many age-related pathologies including Alzheimer's disease (Bonam, Wang et al. 2019). The primary role of lysosomes is to carry out degradation and recycling of damaged proteins and other cellular components. The ability of lysosomes to act in response to multiple signaling inputs involving nutritional status, proteotoxic stress resistance, development and differentiation makes it a critical regulator of organismal survival (Lamming and BarPeled 2019, Savini, Zhao et al. 2019, Villegas, Lehalle et al. 2019). In fact, functional decline in lysosomal activity has been shown to severely affect lifespan and health span in many organisms. Conversely, interventions boosting lysosomal function are emerging as a potent means of promoting lifespan extension and delaying disease pathologies, particularly in relation to the central nervous system (Zhang, Sheng et al. 2009). Survival of neuronal cells that are destined to last throughout the life span relies heavily on lysosomal-based cellular recycling mechanisms. In this regard, identifying novel compounds and strategies to repurpose existing drugs to boost lysosomal function could help delay aging and age-related neurodegeneration.

Potassium restriction as a means to boosts vacuolar acidity and extend lifespan in Sachromyces cerevisiae was recently shown by Sasikumar et al (Sasikumar, Killilea et al. 2019). The lifespan extending effect of potassium restriction were also recapitulated by the supplementation with sodium polystyrene sulfonate (SPS), a potassium-chelating drug. The drug has been in medical use to treat hyperkalemia (high potassium levels) since 1958 (Hagan, Farrington et al. 2016). In the present study, we tested the functional conservation of SPS efficacy in higher model organisms. We specifically investigated effect of SPS supplementation on lysosomal function and proteotoxicity. Our results demonstrate that SPS enhances lysosomal function both in C.elegans and human neuronal cells and suppresses proteotoxicity caused by amyloid- $\beta$ and hyper-phosphorylated-TAU, key drivers of Alzheimer's disease. Overall our work supports the potential use of SPS as a prospective geroprotective intervention with the ability to suppress proteotoxicity caused by neurotoxic proteins and its future testing in preclinical mouse models.

\section{RESULTS}

Earlier work demonstrated that SPS extends lifespan in yeast by boosting vacuolar acidity. We sought to test whether this mechanism of SPS is conserved and if it could also trigger $\mathrm{pH}$ changes in $C$. elegans lysosomes. To explore this idea, we first assayed lysosomal acidity with the dye acridine orange whose emission wavelength shifts when it is sequestered within acidic lysosomes (Moriyama, Takano et al. 1982). Results revealed a 4.5 -fold increase in acridine orange staining in animals treated with SPS relative to untreated controls (Figure 1A). To evaluate the impact of SPS on lysosome function, we used a reporter strain expressing the LGG-1 lysosomal cargo protein fused to mCherry and an acid-inactivated GFP; quenching of GFP signal from this reporter reflects its delivery into the acidic lysosome (Chang, Kumsta et al. 2017). This strain, when treated with SPS, exhibited a 3-fold increase in lysosomal processing of the reporter relative to untreated controls (Figure 1B). For an independent test of lysosomal function, we made use of an assay quantifying sensitivity to the lysosomotropic agent chloroquine (Wibo and Poole 1974). After chronic SPS treatment, animals were 2.5 -fold more resistant to a lethal chloroquine 
dose than untreated controls (Figure 1C). Taken together, these data point to a marked boost in lysosomal acidity and function in $C$. elegans in response to SPS.

On the premise that SPS promotes the degradative function of lysosomes, we expected that this drug would also improve clearance of proteotoxic aggregates. To test this, we used a well-characterized $C$. elegans disease model in which expression of the aggregation-prone $A \beta_{1-42}$ fragment of the human amyloid precursor protein in body wall muscle leads to temperature-dependent paralysis (McColl, Roberts et al. 2012). In this strain, SPS treatment conferred a 3-fold resistance to paralysis relative to controls (Figure 1D). We concluded that SPS helps resolve proteotoxic stress, mirroring the increased lysosomal function in these animals. Decline in lysosomal function and inability to maintain $\mathrm{pH}$ gradient is associated with accelerated aging in C.elegans (Anand, Holcom et al. 2020, Sun, Li et al. 2020). Thus, we tested ability of SPS to delay aging and extend lifespan in C. elegans. We observed WT animals treated with $10 \mathrm{mM}$ SPS throughout adulthood lived $13 \%$ longer than controls (Figure 1E). We did not observe any general toxic effects of the SPS as there was no changes in the fecundity i.e. total number of eggs hatched (Fig. S1). Control experiments revealed no consistent effects of SPS on body movement (Figure S2), arguing against a feeding defect as implicated in the longevity of animals with compromised potassium transport (Davis, Somerville et al. 1995, Hamilton, Dong et al. 2005). Together, our data show that SPS has measurable health benefits in $C$. elegans in the context of physiology and behavior as well as increased viability into old age.

We next tested the ability of SPS to boost lysosomal function in mammalian neuronal cells. We exposed human $\mathrm{H} 4$ neuronal cells to SPS for 6 hours and quantified mRNA expression of lysosomal function-specific genes. Treatment of cells with 10 mM SPS significantly enhanced expression of several key lysosomal-specific genes up to levels of 1.5 - 2-fold, suggesting an increase in lysosomal function (Figure 2A). To further evaluate lysosomal function, we monitored the turnover rate of LC3-I, the mammalian homolog of $C$. elegans LGG-1, in SPS-treated neuronal cells both in the presence and absence of the lysosomal V-ATPase inhibitor bafilomycin A1. We observed that samples treated with SPS displayed increases in LC3-II levels which were further enhanced in the presence of bafilomycin $\mathrm{A} 1$ (Figure $2 \mathrm{~B}$ and $2 \mathrm{C}$ ). These results demonstrate that SPS treatment enhances lysosomal processing of LC3-II, confirming enhanced autophagy flux and lysosomal function. We further verified lysosomal function by performing a Dye Quenched-Bovine Serum Albumin (DQBSA) based assay (Marwaha and Sharma 2017). DQ-BSA is a heavily labeled fluorescent substrate of the lysosomal proteases, which shows reduced fluorescence under basal conditions due to a strong quenching effect. Upon hydrolysis of the DQBSA to single, dye-labeled peptides by lysosomal proteases, quenching is prevented, producing brightly fluorescent products. We observed that SPS-treated rat N27 neuronal cells showed a dose-dependent increase in fluorescence intensity (Figure 2D). This increase in fluorescence intensity was completely suppressed by the lysosomal inhibitor bafilomycin A1 (Figure 2D), demonstrating SPS enhances lysosomal function in mammalian neuronal cells. To test our hypothesis that SPSenhanced lysosomal function suppresses proteotoxicity, we utilized human neuronal SH-SY5Y cells expressing full-length 4R-isoform of TAU. We found that SPS treatment reduced the level of aggregation associated phosphorylated-TAU (Figure $2 \mathrm{E}$ and $2 \mathrm{~F}$ ). Taken together, these results suggest a conserved function of the 
potassium chelator SPS in human neuronal cells and its therapeutic potential in suppressing proteotoxicity.

\section{DISCUSSION}

Our work demonstrates a previously unknown role for SPS in suppressing proteotoxicity caused by human neurotoxic proteins. The conserved role of SPS in enhancing lysosomal function in yeast, worms and human neuronal cells strongly suggests its likelihood of working in higher in vivo models. These findings are in line with other published work showing many potent anti-aging interventions are closely related to enhanced lysosomal function (Carmona-Gutierrez, Hughes et al. 2016). The lifespan-extending property of SPS further implies that the protective effect of SPS could potentially extend beyond suppression of proteotoxicity to delaying aging. The significance of our work is broad and is of general interest considering SPS is already used as a medication in humans and has been on the market for more than 60 years.

Although our study establishes that SPS enhances lysosomal function, it's still not clear how exactly it does so. Due to its ability to disrupt ionic imbalance across the lysosomal membrane, SPS likely leads to lysosomal acidification. In mammalian cells the $\mathrm{Na}^{+} / \mathrm{K}^{+}$ATPase pump regulates the sodium gradient across the membrane, which in turn drives the $\mathrm{Na}^{+} / \mathrm{H}^{+}$antiporter and thus cellular $\mathrm{pH}$ (Kaplan 2002). SPS is a cross-linked polymer with a reactive sulfonic group that exchanges existing sodium $\left(\mathrm{Na}^{+}\right)$for potassium $\left(\mathrm{K}^{+}\right)$cations. This could potentially lead to a decrease in potassium concentration with simultaneous increases in the sodium concentration upon SPS exposure (Nepal, Bucaloiu et al. 2010). Overall, these changes by SPS profoundly affect lysosomal $\mathrm{pH}$ and function, imparting beneficial effects. Previous work in yeast cells shows that similar effects can be recapitulated by decreasing potassium concentration in the media, while no changes were observed by sodium supplementation, thus arguing in favor of potassium restriction as a potential mechanism resulting in a boost of lysosomal pH (Sasikumar, Killilea et al. 2019). Also, a recent work show that potassium starvation induces autophagy in yeast (Rangarajan, Kapoor et al. 2020), which falls in line with our observations, although these studies still needs to be validated in other organisms. Nevertheless, the idea of utilizing pharmacological compounds that alter $\mathrm{Na}^{+} / \mathrm{K}^{+}$ionic imbalances to target neurodegenerative diseases is fairly novel and relatively untested. This is particularly interesting since several neurodegenerative diseases including Alzheimer's have been reported to have a significant $\mathrm{Na}^{+} / \mathrm{K}^{+}$ionic imbalance (Vitvitsky, Garg et al. 2012). Future experiments quantifying concentration of different ions in response to SPS treatment will be important to help decipher how SPS effect's lysosomal function and suppresses proteotoxicity. 

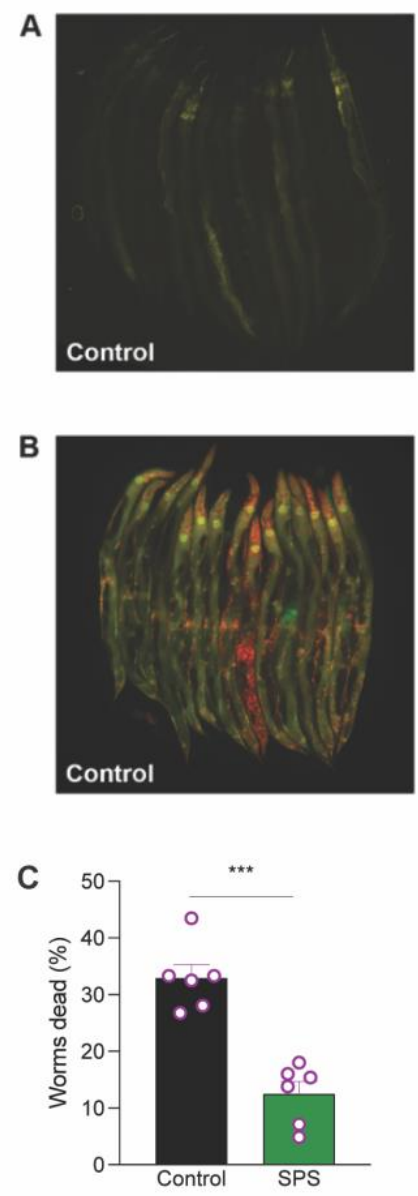
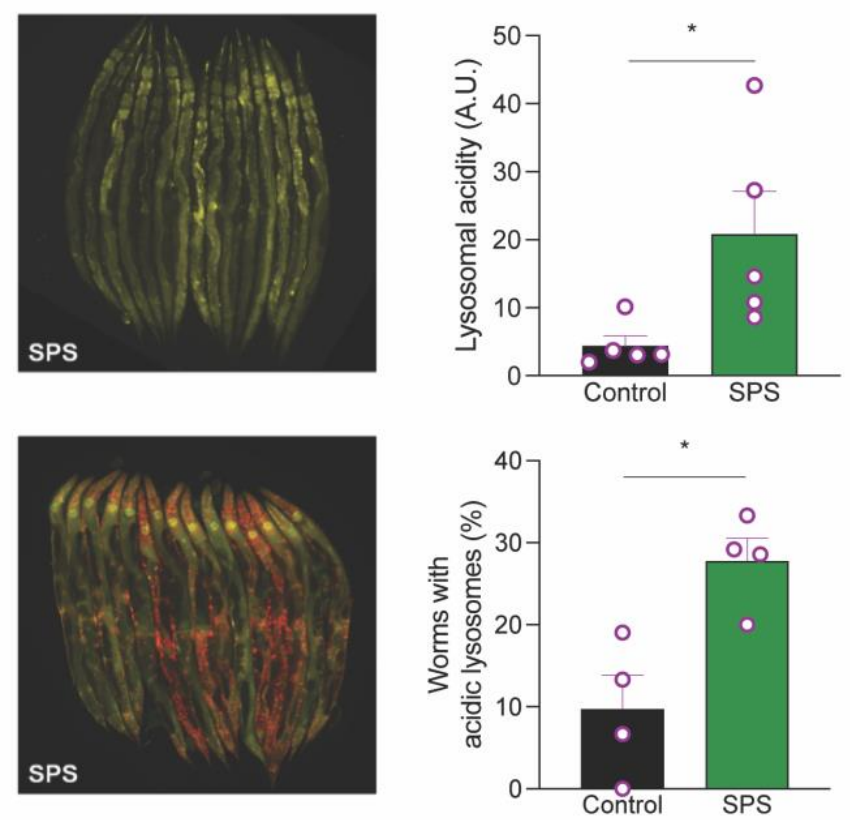

D

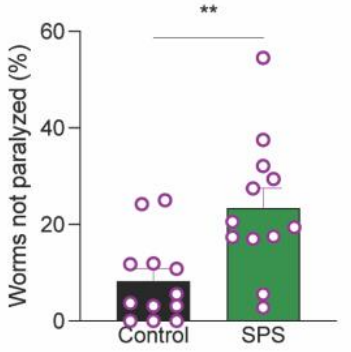

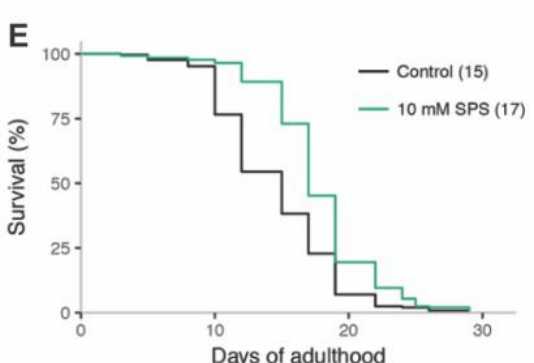

Figure 1. Sodium polystyrene sulfonate (SPS) treatment of worms improves pHdependent processing of lysosomal cargo, enhances proteotoxic stress resistance and extends lifespan in C. elegans. (A), Left, representative fluorescence microscopy images of wild-type worms maintained in the absence (control) or presence of $10 \mathrm{mM}$ SPS and then treated with acridine orange to stain acidic lysosomes. Right, each bar reports mean acridine orange intensity per worm ( $n=5$ replicates, 15 worms per replicate) in arbitrary units (A.U.). (B), Left, representative fluorescence microscopy images of worms maintained in the absence (control) or presence of $10 \mathrm{mM} \mathrm{SPS}$ expressing a fusion of the lysosomal cargo protein LGG-1 to mCherry and acid-inactivated GFP. Right, each bar reports the proportion of worms ( $n=4$ replicates, 15 worms per replicate) expressing the LGG-1 reporter that exhibited reduced GFP intensity relative to mCherry, reflecting processing of the reporter in the acidic lysosome. (C), Each bar reports the mean viability ( $n$ $=6$ replicates, 50 worms per replicate) of wild-type worms treated with the lysosomotropic agent chloroquine $(20 \mathrm{mM})$, after maintenance in $10 \mathrm{mM}$ SPS or control treatment. (D), Each bar reports the mean proportion ( $n=12$ replicates, 35 worms per replicate) of amyloid- $\beta$ expressing worms maintained in the absence (control) or presence of $10 \mathrm{mM}$ SPS that exhibited no detectable paralysis. Error bars report standard error. ${ }^{* * *}$, one-tailed $t$-test $p<$ $0.001 ;{ }^{*}, p<0.01 ;{ }^{*}, p<0.05$; tests were paired for $A$ and $B$ and unpaired for $C$ and $D$. (E), Shown are results from a representative trial of a lifespan assay of wild-type $C$. elegans hermaphrodites on NGM plates (control) or NGM plates supplemented with $10 \mathrm{mM}$ SPS. Numbers in parentheses report median lifespan in days $(n=262$ for control, $n=268$ for SPS). Significance of a Cox proportional hazards test comparing the two conditions was $p=$ $3.5 \times 10^{-11}$. Results from additional trials are reported in the supplementary table 1 . 
A

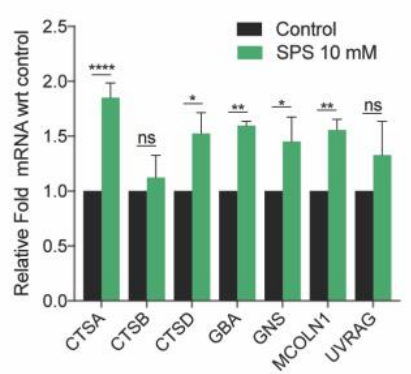

D

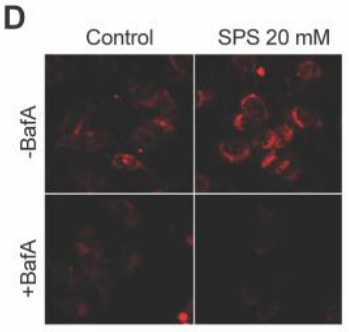

B

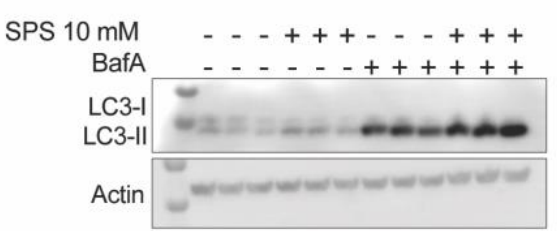

$\mathbf{F}$

G

C

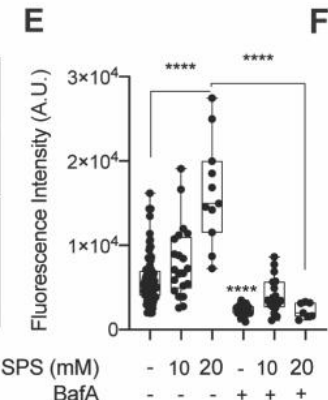

SPS (mM)

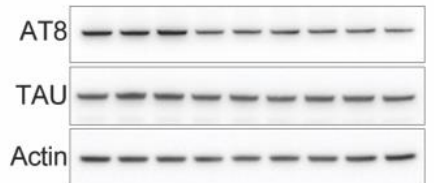

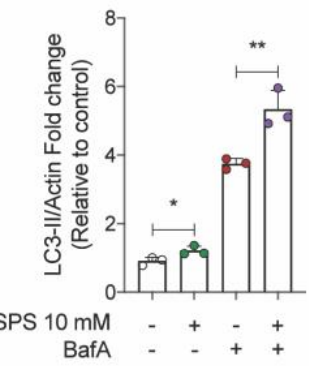

- Control -SPS $10 \mathrm{mM}$ - SPS $20 \mathrm{mM}$

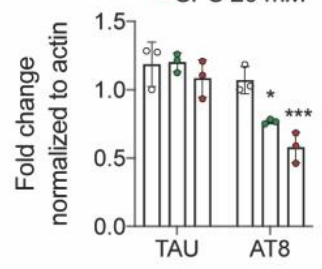

Figure 2. Sodium polystyrene sulfonate (SPS) boosts lysosomal function in mammalian neuronal cells and reduces level of aggregation-associated

phosphorylated-TAU. (A), Fold mRNA levels of lysosomal-specific genes in $10 \mathrm{mM}$ SPStreated human $\mathrm{H} 4$ neuronal cells relative to control samples (no SPS). Graph represents the mean \pm SD of two biological repeats. $p$-values calculated using two-way ANOVA with Sidak's multiple comparison test. (B), Levels of LC3-I and LC3-II protein in $10 \mathrm{mM}$ SPStreated $\mathrm{H} 4$ neuronal cells in the absence and presence of the lysosomal V-ATPase inhibitor bafilomycin $A 1$ ( $n=3$ per condition). (C), Fold changes in levels of normalized LC3-II protein relative to control sample (no SPS) quantified using NIH image J software. Graph represents mean fold intensity \pm SD of Quantification of band intensity. $p$-values calculated using unpaired Student's $t$-test. (D), Represntative image of Dye Quenched-Bovine Serum Albumin (DQ-BSA) stained rat N27 neuronal cells. Images were taken in Axio Zeiss microscope using RFP filter. (E), Graph represents mean fluorescence intensity \pm SD of SPS treated (10 and $20 \mathrm{mM}$ ) rat N27 dopaminergic cells with and without addition of the lysosomal V-ATPase inhibitor bafilomycin A1. Each dot represents individual cell $(n=3)$. $p$ values calculated using one-way ANOVA with Tukey's multiple comparison test. (F), Levels of TAU and phosphorylated TAU (Ser202, Thr205)/AT8 in 10 and 20 mM SPS-treated TAU expressing human SH-SY5Y neuronal cells ( $n=3$ per condition). (G), Fold changes in levels of normalized TAU and phosphorylated-TAU relative to control sample (no SPS) quantified using NIH image $\mathrm{J}$ software. Graph represents mean fluorescence intensity \pm SD of SPS treated (10 and $20 \mathrm{mM}$ ) TAU expressing SH-SY5Y neuronal cells. $p$-values calculated using two-way ANOVA with Tukey's multiple comparison test. $p$-values represents $p<0.001 ;{ }^{* *}, p$ $<0.01 ;{ }^{*}, p<0.05$ 
bioRxiv preprint doi: https://doi.org/10.1101/2021.10.21.465344; this version posted October $22,2021$. The copyright holder for this preprint (which was not certified by peer review) is the author/funder, who has granted bioRxiv a license to display the preprint in perpetuity. It is made available under aCC-BY-NC-ND 4.0 International license.

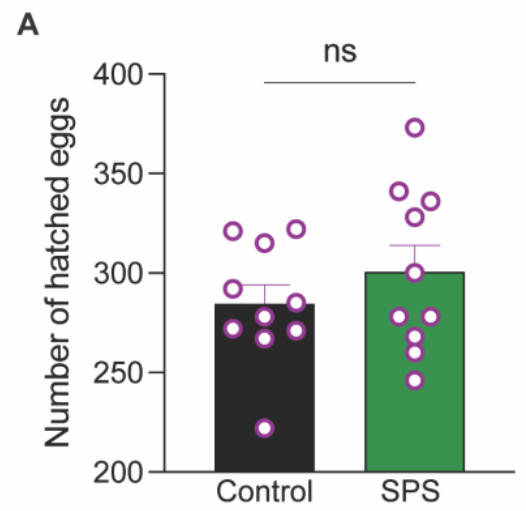

Supplementary Figure 1: No changes in the fecundity upon SPS treatment. Total fecundity quantification i.e., total number of hatched eggs in N2 treated control or $10 \mathrm{mM}$ SPS. $p$-value calculated using an unpaired Student's t-test 
A

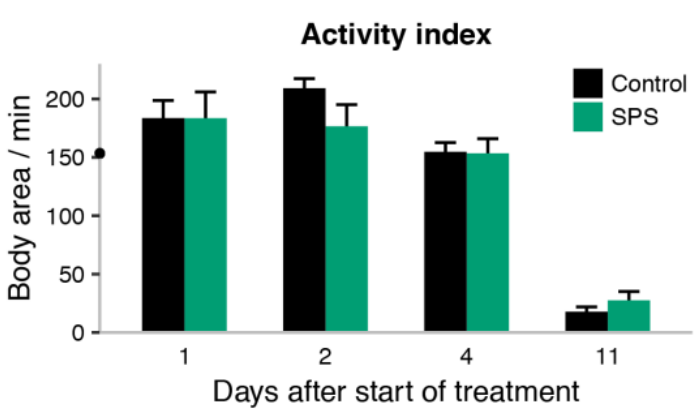

C

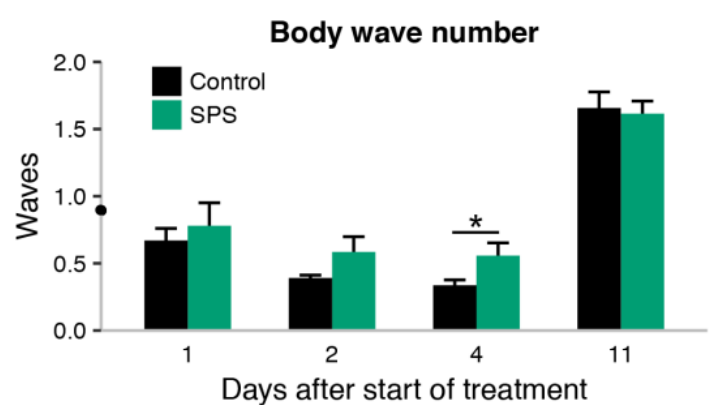

E

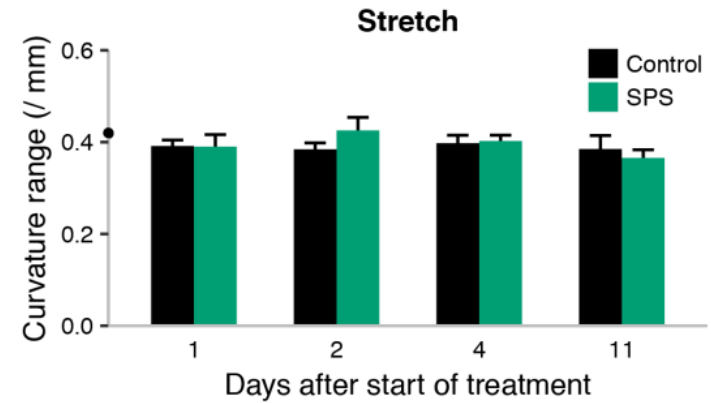

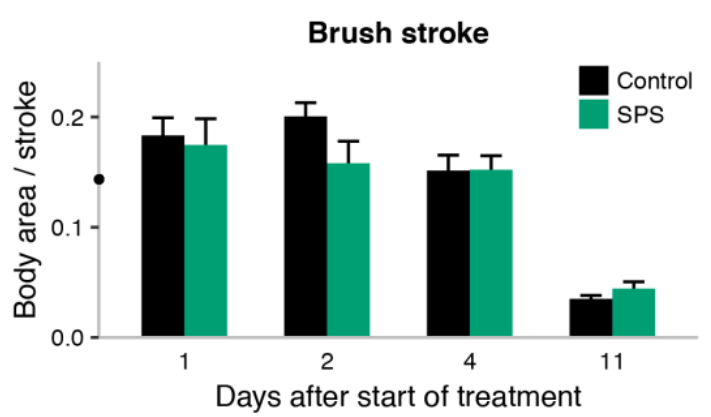

D

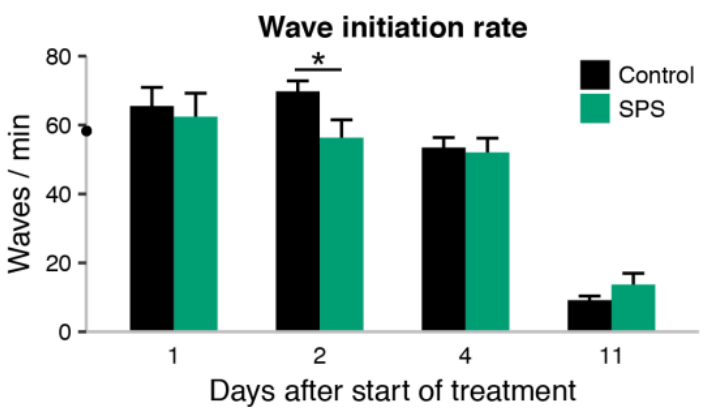

$\mathbf{F}$

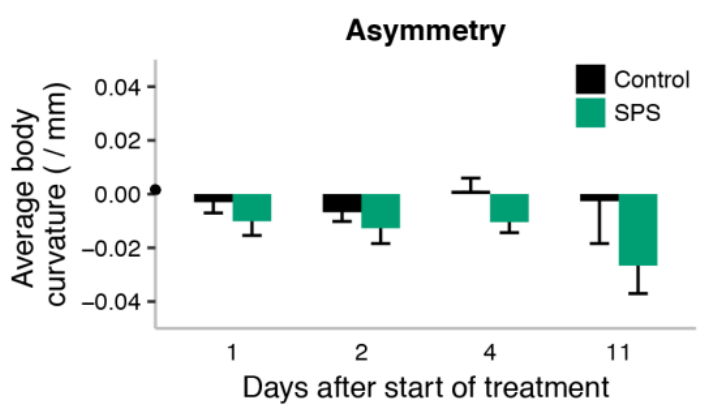

Supplementary Figure 2: SPS has a negligible effect on worm movement. Each panel reports one attribute of the movement of wild-type worms maintained in the absence (control) or presence of $10 \mathrm{mM}$ SPS over the indicated time course starting at day 1 of adulthood. (A), The $y$-axis reports the number of pixels covered by a given animal over the time it took to undertake two swimming strokes. (B), The $y$-axis reports the number of pixels covered by a given animal during a single swimming stroke. (C), The $y$-axis quantifies the sinusoidal movement of the animals. Low scores (between $0-1$ ) indicate that a single wave is propagating across the animal; higher scores indicate less coordinated movement. (D), The $y$-axis reports the number of initiated body bends per minute. (E), The $y$-axis reports the depth of bending of the swimming animal. (F), The $y$-axis reports how balanced the swim posture was per stroke. In a given panel, each bar height reports the mean value of the respective measurement in worms of the respective treatment and timepoint, and error bars report standard error; the black point on the $y$-axis reports the mean value among 1-day-old adult animals pre-treatment. ${ }^{*}, t$-test $0.01 \leq p \leq 0.05$. 


\begin{tabular}{|c|c|c|c|c|}
\hline Trial & Treatment & No. of worms & $\begin{array}{c}\text { Median lifespan } \\
\text { (days) }\end{array}$ & $\boldsymbol{p}$ \\
\hline 1 & Control & 181 & 17 & $2.30 \mathrm{E}-02$ \\
\hline 2 & SPS & 57 & 19 & $3.60 \mathrm{E}-11$ \\
\hline 3 & Control & 249 & 14 & $1.90 \mathrm{E}-11$ \\
\hline
\end{tabular}

Supplementary Table 1: Sodium polystyrene sulfonate (SPS) reproducibly extends worm lifespan. Each row reports results from one trial of lifespan assays of wild-type C.elegans on NGM plates (control) or NGM plates supplemented with $10 \mathrm{mM}$ SPS. The last column reports results of a Cox proportional hazards test comparing the lifespans of treated and untreated animals in the respective trial. 


\section{EXPERIMENTAL PROCEDURES}

Nematode culture, strains, and maintenance. Except where noted below, $C$. elegans hermaphrodites were maintained on nematode growth medium (NGM) agar plates seeded with E. coli bacterial strain OP50 at $20^{\circ} \mathrm{C}$ as described previously (Brenner 1974). Strains used in this study were wildtype Bristol N2; GMC101 dvls100 [unc-54p::A-beta-1-42::unc-54 3'-UTR + mtl-2p::GFP]; and MAH215 sqls11 [lgg1p::mCherry::GFP::Igg-1 + rol-6] rollers.

Worm sodium polystyrene sulfonate (SPS) treatment. A $500 \mathrm{mM}$ stock of SPS was prepared in sterile water and stored at $4^{\circ} \mathrm{C}$ for up to 10 days. From the stock solution, $130 \mu \mathrm{l}$ of the working SPS solution $(10 \mathrm{mM})$ was prepared by mixing $60 \mu \mathrm{L}$ of stock solution with $70 \mu \mathrm{L}$ of sterile water, and was added to the top of the $35 \mathrm{~mm}$ NGM plates (3 mL NGM agar) already seeded with a bacterial OP50 lawn. SPS was distributed over the entire plate surface and allowed to dry in a sterile hood with the lid open for at least 1 hour. Plates were then allowed to sit at $20^{\circ} \mathrm{C}$ for 24 hours before use or before moving into $4^{\circ} \mathrm{C}$ for storage up to 2 weeks. For all worm experiments, animals were treated as follows except where noted below: a synchronous population was obtained by a 2-hour egg lay from gravid adult hermaphrodites, after which the adults were removed and the eggs were left to develop into adults at $20^{\circ} \mathrm{C}$. On their first day of adulthood, the population was split into two, with half transferred to $35 \mathrm{~mm}$ NGM assay plates containing $10 \mathrm{mM}$ SPS and the other half transferred to control plates.

Worm lifespan assay. Wild-type worms were synchronized and SPS-treated on day 1 of adulthood as above, except that following day 1, transfers continued periodically from each respective plate to a fresh plate of the same formulation (10 mM SPS or no-drug control) to ensure ample supply of drug and food. Survival was quantified by counting dead and live worms once every two or three days. We refer to the pipeline from egg lay through treatment and survival quantification as a trial, with a given such experiments were performed using drug-treated and control conditions in parallel and 35-40 worms on each of at least 3 replicate plates for each condition; three such independent trials were performed.

Worm movement assay. Wild-type worms were synchronized and SPS-treated on day 1 of adulthood as above, except that following day 1, transfers continued periodically from each respective plate to a fresh plate of the same formulation (SPS or no-drug control) to ensure ample supply of drug and food. After $24 \mathrm{~h}, 48 \mathrm{~h}, 96 \mathrm{~h}$ or $264 \mathrm{~h}$, worms were removed from their agar plate cultures and transferred into a swimming buffer. 30-second videos were then immediately captured of the swimming animals, which were then removed from the study and subjected to the CeleST analysis pipeline (Restif, Ibanez-Ventoso et al. 2014). 20 animals were captured for each time point and condition.

Total fecundity quantification. The average number of fertilized eggs (fecundity) laid by worms was determined by transferring individual L4 larval staged wild-type worms onto NGM agar treated control or SPS bacterial plates $(n=9-10$ worms per condition). Worms were allowed to lay eggs for the next 24 hours and then transferred to a fresh treated NGM agar bacterial plate. Worms were subsequently moved to fresh plates every day until they ceased laying eggs. On each plate, the 
total numbers of eggs laid and hatched were scored. The graph represents number of eggs hatched in each condition.

Worm acridine orange (AO) staining. Wild-type worms were synchronized and SPS-treated on day 1 of adulthood as above, except that both SPS and control plates were supplemented with $10 \mu \mathrm{g} / \mathrm{mL}$ 5-fluoro-2'-deoxyuridine (FUdR) to inhibit progeny production. On day 2 of adulthood, worms were transferred from their respective plate to a fresh plate of the same formulation (SPS or no-drug control). On day 4, worms from these plates were transferred using a platinum pick to a $20 \mu \mathrm{l}$ drop of $\mathrm{AO}(0.05 \mathrm{mg} / \mathrm{ml}$ in S-basal buffer, http://www.wormbook.org/chapters/www_strainmaintain/strainmaintain.html) on the side of a $2 \%$ agarose pad slide and allowed to stain for 15 minutes. After 15 minutes, the $\mathrm{AO}$ solution was removed using a pipette, and worms were washed with S-basal buffer. $\sim 15$ worms were then transferred to a drop of fresh S-basal buffer on the agarose pad, to which was added $5 \mu \mathrm{l}$ of $2 \mathrm{mM}$ levamisole to immobilize. Worms were visualized under a rhodamine filter on a Zeiss fluorescence microscope. Intensity in a region delimited manually from the perimeter of each worm was quantified in ImageJ. The pipeline from growth through treatment, staining and quantification we refer to as a replicate, with a given such experiments were performed using drug-treated and control conditions in parallel; five such independent replicates were performed.

Tandem-tagged LGG-1 reporter imaging: MAH215 worms were synchronized and SPS-treated on day 1 of adulthood as above. After two hours on SPS or no-drug control plates, 15 worms were imaged using GFP and rhodamine filters on a Zeiss fluorescence microscope. GFP and mCherry intensity from individual worms were quantified using ImageJ. For each worm, we tabulated the ratio of mCherry and GFP intensities and, as a metric of LGG-1 processing, we tabulated the proportion of worms where this ratio exceeded 1.5. The pipeline from growth through treatment, staining, and quantification we refer to as a replicate, with a given that such experiments were performed using drug-treated and control conditions in parallel; four such independent replicates were performed.

Chloroquine toxicity assay: Wild-type worms were synchronized and SPS-treated on day 1 of adulthood as above, except that following day 1, transfers continued periodically from each respective plate to a fresh plate of the same formulation (SPS or no-drug control) to ensure ample supply of drug and food. On day 7, worms from each plate were transferred to $20 \mathrm{mM}$ chloroquine supplemented NGM agar plates without SPS. On day 9 and day 11, worms were transferred to fresh chloroquine plates. On day 11, viability was quantified by counting dead and live worms. We refer to the pipeline from egg lay through SPS treatment, chloroquine treatment, and survival quantification as a trial, with a given such experiments were performed using SPS-treated and control conditions in parallel using $\sim 50$ worms on each of 3 replicate plates for each condition. Two such independent trials were performed.

Worm amyloid-beta paralysis assay. A synchronous population of GMC101 worms was obtained by 2 -hour egg-lay at $20^{\circ} \mathrm{C}$. At the L4 stage, 48 -hours after egglay, the population was split into two, with half transferred to plates containing $10 \mathrm{mM}$ SPS and the other half to control plates; all were incubated at $25^{\circ} \mathrm{C}$ for an additional 48 hours. At this timepoint, body movement was assessed as follows. A given worm 
was scored as paralyzed if it (1) failed to complete a full body movement, i.e a point of inflection traversing the entire body length, either spontaneously or touchprovoked using platinum wire, and (2) exhibited a halo of cleared bacteria around its head, indicative of insufficient body movement to access food. We refer to the pipeline from egg lay through SPS treatment and paralysis scoring as a trial, with a given that such experiments were performed using SPS-treated and control conditions in parallel and $\sim 35$ worms on each of 4 replicate plates for each condition. Three such independent trials were performed.

Cell culture and maintenance. Human H4 neuroglioma cells (ATCC) were maintained in Dulbecco's Modified Eagle Medium (DMEM), human SH-SY5Y-GFPTau neuroblastoma cells (ATCC) were maintained in HyClone Classical Liquid DMEM-F12 media, and rat N27 dopaminergic neural cells (Millipore) were maintained in Roswell Park Memorial Institute (RPMI) 1640 medium. All culture media were supplemented with 10\% FBS (Serum Plus - II, Corning) and $1 \%$ penicillin-streptomycin (Corning). Cells were incubated at $37^{\circ} \mathrm{C}$ in a $5 \% \mathrm{CO}_{2}$ humidified cell culture incubator.

Chemicals and buffers. Sodium polystyrene sulfonate (SPS) (Sigma) was dissolved in the appropriate basal media and stored at $4^{\circ} \mathrm{C}$ for up to 10 days at a concentration of 1M. Dulbecco's phosphate-buffered saline (DPBS) was purchased from Corning. PBST buffer (PBS plus $0.1 \%$ Tween-20) was used for blot washing. Blocking buffer and primary antibody incubation solution was $5 \%$ non-fat dairy milk powder (VWR) or $5 \%(\mathrm{w} / \mathrm{v})$ BSA (Sigma) for phospho-antibodies.

Cellular SPS treatment. The working SPS solution (10 mM) was prepared by mixing stock solution in complete media at a dilution of 1:100 and adding to adherent cells already seeded at the proper density in tissue culture plates. Cells were treated with SPS for 4-6 hours, with some samples being pretreated with bafilomycin-A1 (Sigma-Aldrich) for 1 hour at $100 \mathrm{nM}$.

RNA isolation and qPCR. H4 cells were treated with SPS as described and collected for RNA isolation. RNA was isolated using a Zymo Research Quick-RNA miniprep kit (Zymo). Quality and concentration of RNA was confirmed on a NanoDrop 2000 spectrophotometer (Thermo). From the RNA isolation, 1 ug was used to generate cDNA using a High-Capacity cDNA Reverse Transcriptase kit (Applied Biosystems). The qPCR was performed using $20 \mathrm{ng}$ of cDNA product per reaction in the presence of Lightcycler 480 SYBR green dye (Roche). Lysosomal gene primers were designed using PrimerBLAST and ordered from Eurofins; they were used at a subsequent working concentration of $200 \mathrm{nM}$. The sequence of forward $(F)$ and reverse $(R)$ of the primers (5'-3') used are: CTSA-

F:CAGGCTTTGGTCTTCTCTCCA, CTSA-R: TCACGCA TTCCAGGTCTTTG, CTSB-F: AGTGGAGAATGGCACACCCTA, CTSB-R:

AAGAAGCCATTGTCACCCCA, CTSD-F: AACTGCTGGACATCGCTTGCT, CTSDR: CATTCTTCACGTAGGTGCTGGA, GBA-F: TGGGTACCCGGATGATGTTA, GBA-R: AGATGCTGCTGCTCTCAACA, GNS-F: CCCATTTTGAGAGGTGCCAGT, GNS-R: TGACGTTACGGCCTTCTCCTT, MCOLN1-F:

TTGCTCTCTGCCAGCGGTACTA, MCOLN1-R: GCAGTCAGTAACCACCATCGGA, UVRAG-F: CATCTGTGTCTTGTTTCGTGG, UBRAG-R: TTCA

TTTTGGTTTCGGGCATG 
DQ Red assay. N27 cells were seeded in a 4-well chamber slide at 30,000 cells per well. The next day, DQ Red BSA dye (ThermoFisher Scientific) was prepared at a working of concentration $10 \mu \mathrm{g} / \mathrm{ml}$ (1:200 dilution) in N27 media. Cells were washed once in pre-warmed DPBS, then DQ dye was added to cells followed by incubation for 1 hour. The DQ media was then removed and replaced with media +/bafilomycin. After 1 hour, SPS was added at a final concentration of $10 \mathrm{mM}$ for 4 hours. Cells were then fixed with 4\% PFA prepared in DPBS for 10 minutes. Slides were mounted with Prolong Gold Antifade Mountant in the presence of DAPI (ThermoFisher Scientific). Images were taken on a confocal microscope (Zeiss) at 40x magnification.

Western blotting. Cells treated with SPS either alone or in conjunction with bafilomycin-A1 were collected and subsequently lysed in lysis buffer $(50 \mathrm{mM}$ Tris$\mathrm{HCl} \mathrm{pH} \mathrm{8,150} \mathrm{mM} \mathrm{NaCl}, 1 \% \mathrm{NP}-40$ ) supplemented with PhosStop phosphatase inhibitor cocktail (Sigma Aldrich) and protease inhibitor cocktail (Sigma Aldrich). Protein concentrations were quantified by Bradford assay per manufacturer's instructions (BioRad). Samples were normalized per protein via dilution in lysis buffer and SDS protein dye. Samples were heated at $90^{\circ} \mathrm{C}$ for 15 minutes and then centrifuged at $15,000 \mathrm{xg}$ for 10 minutes. Samples were next electrophoresed on a NuPAGE $4-12 \%$ Bis-Tris gel (ThermoScientific), the resulting gel transferred to PVDF membrane, blocked in blocking buffer (5\% milk in PBST) and probed with primary and then secondary antibodies. The primary antibodies used were $p$-Tau (S422) from ThermoFisher (44-764G), p-Tau (S204, T205) from ThermoFisher (MN1020), Tau from ThermoFisher (MN1000), LC3B from Cell Signaling Technologies (2775S), and $\beta$-actin from Cell Signaling Technologies (3700S).

\section{ACKNOWLEDGEMENTS}

The authors thank Dr. Arjun N. Sasikumar for helpful discussion. This work was supported by NIH RF1 AG057358 to JKA. The C. elegans strains used in this work were provided by the Caenorhabditis Genetics Center (CGC), funded by the NIH Office of Research Infra- structure Programs (P40OD010440). MC is supported by the postdoctoral fellowship from the Larry L. Hillblom Foundation.

\section{AUTHOR CONTRIBUTIONS}

Conception and design, R.B.B., M.C. and J.K.A.; Data acquisition and analysis, C.A., M.L., A.R., M.S., T.G., A.F., M.B., E.B. and M.C.; Writing -Review \& Editing, R.B.B., M.C., G.J.L. and J.K.A.; Supervision, M.C. and J.K.A.; Funding Acquisition, G.J.L. and J.K.A

\section{CONFLICT OF INTEREST}

None

\section{DATA AVAILABILITY STATEMENT}

The data that support the findings of this study are available from the corresponding author upon reasonable request. 


\section{REFERENCES}

Anand, N., A. Holcom, M. Broussalian, M. Schmidt, S. J. Chinta, G. J. Lithgow, J. K. Andersen and M. Chamoli (2020). "Dysregulated iron metabolism in C. elegans catp6/ATP13A2 mutant impairs mitochondrial function." Neurobiol Dis 139: 104786. Bonam, S. R., F. Wang and S. Muller (2019). "Lysosomes as a therapeutic target." Nat Rev Drug Discov 18(12): 923-948.

Brenner, S. (1974). "The genetics of Caenorhabditis elegans." Genetics 77(1): 7194.

Carmona-Gutierrez, D., A. L. Hughes, F. Madeo and C. Ruckenstuhl (2016). "The crucial impact of lysosomes in aging and longevity." Ageing Res Rev 32: 2-12.

Chang, J. T., C. Kumsta, A. B. Hellman, L. M. Adams and M. Hansen (2017). "Spatiotemporal regulation of autophagy during Caenorhabditis elegans aging." Elife 6.

Davis, M. W., D. Somerville, R. Y. Lee, S. Lockery, L. Avery and D. M. Fambrough (1995). "Mutations in the Caenorhabditis elegans Na,K-ATPase alpha-subunit gene, eat-6, disrupt excitable cell function." J Neurosci 15(12): 8408-8418.

Hagan, A. E., C. A. Farrington, G. C. Wall and M. M. Belz (2016). "Sodium polystyrene sulfonate for the treatment of acute hyperkalemia: a retrospective study." Clin Nephrol 85(1): 38-43.

Hamilton, B., Y. Dong, M. Shindo, W. Liu, I. Odell, G. Ruvkun and S. S. Lee (2005). "A systematic RNAi screen for longevity genes in C. elegans." Genes Dev 19(13): 1544-1555.

Kaplan, J. H. (2002). "Biochemistry of Na,K-ATPase." Annu Rev Biochem 71: 511 535.

Lamming, D. W. and L. Bar-Peled (2019). "Lysosome: The metabolic signaling hub." Traffic 20(1): 27-38.

Marwaha, R. and M. Sharma (2017). "DQ-Red BSA Trafficking Assay in Cultured Cells to Assess Cargo Delivery to Lysosomes." Bio Protoc 7(19).

McColl, G., B. R. Roberts, T. L. Pukala, V. B. Kenche, C. M. Roberts, C. D. Link, T. M. Ryan, C. L. Masters, K. J. Barnham, A. I. Bush and R. A. Cherny (2012). "Utility of an improved model of amyloid-beta (Abeta(1)(-)(4)(2)) toxicity in Caenorhabditis elegans for drug screening for Alzheimer's disease." Mol Neurodegener 7: 57. Moriyama, Y., T. Takano and S. Ohkuma (1982). "Acridine orange as a fluorescent probe for lysosomal proton pump." J Biochem 92(4): 1333-1336.

Nepal, M., I. D. Bucaloiu and E. R. Norfolk (2010). "Hypernatremia in a patient treated with sodium polystyrene sulfonate." Int J Nephrol Renovasc Dis 3: 141-143. Rangarajan, N., I. Kapoor, S. Li, P. Drossopoulos, K. K. White, V. J. Madden and H. G. Dohlman (2020). "Potassium starvation induces autophagy in yeast." J Biol Chem 295(41): 14189-14202.

Restif, C., C. Ibanez-Ventoso, M. M. Vora, S. Guo, D. Metaxas and M. Driscoll (2014). "CeleST: computer vision software for quantitative analysis of C. elegans swim behavior reveals novel features of locomotion." PLoS Comput Biol 10(7): e1003702.

Sasikumar, A. N., D. W. Killilea, B. K. Kennedy and R. B. Brem (2019). "Potassium restriction boosts vacuolar acidity and extends lifespan in yeast." Exp Gerontol 120: 101-106.

Savini, M., Q. Zhao and M. C. Wang (2019). "Lysosomes: Signaling Hubs for Metabolic Sensing and Longevity." Trends Cell Biol 29(11): 876-887. 
Sun, Y., M. Li, D. Zhao, X. Li, C. Yang and X. Wang (2020). "Lysosome activity is modulated by multiple longevity pathways and is important for lifespan extension in C. elegans." Elife 9.

Villegas, F., D. Lehalle, D. Mayer, M. Rittirsch, M. B. Stadler, M. Zinner, D. Olivieri, P. Vabres, L. Duplomb-Jego, E. De Bont, Y. Duffourd, F. Duijkers, M. Avila, D.

Genevieve, N. Houcinat, T. Jouan, P. Kuentz, K. D. Lichtenbelt, C. Thauvin-Robinet, J. St-Onge, J. Thevenon, K. L. I. van Gassen, M. van Haelst, S. van

Koningsbruggen, D. Hess, S. A. Smallwood, J. B. Riviere, L. Faivre and J. Betschinger (2019). "Lysosomal Signaling Licenses Embryonic Stem Cell

Differentiation via Inactivation of Tfe3." Cell Stem Cell 24(2): 257-270 e258.

Vitvitsky, V. M., S. K. Garg, R. F. Keep, R. L. Albin and R. Banerjee (2012). "Na+ and $\mathrm{K}+$ ion imbalances in Alzheimer's disease." Biochim Biophys Acta 1822(11): 1671-1681.

Wibo, M. and B. Poole (1974). "Protein degradation in cultured cells. II. The uptake of chloroquine by rat fibroblasts and the inhibition of cellular protein degradation and cathepsin B1." J Cell Biol 63(2 Pt 1): 430-440.

Zhang, L., R. Sheng and Z. Qin (2009). "The lysosome and neurodegenerative diseases." Acta Biochim Biophys Sin (Shanghai) 41(6): 437-445. 